\title{
AiMT
}

Advances in Military Technology

Vol. 14, No. 2 (2019), pp. 245-261

ISSN 1802-2308, eISSN 2533-4123

DOI 10.3849/aimt.01295

\section{Prediction of Vortex Induced Aerodynamic Noise from Wind Turbine Blades}

\author{
V. Bhargava ${ }^{1 *}$, S.P. Maddula ${ }^{2}$ and R. Samala ${ }^{3}$ \\ ${ }^{1}$ GE Global Research, JFWTC, Whitefield, Bangalore, India. \\ ${ }^{2}$ Department of Mechanical Engineering, GITAM School of Technology, Hyderabad, India \\ ${ }^{3}$ Department of Applied Mechanics, Indian Institute of Technology, Madras, Chennai, India
}

The manuscript was received on 14 February 2019 and was accepted after revision for publication on 31 August 2019.

\begin{abstract}
:
An important aerodynamic noise source from lifting surface occurs from trailing edge of an aerofoil as found in wind turbine blades. In this work, semi-empirical method proposed by Brookes, Pope, Marcolini is applied to evaluate trailing edge bluntness vortex shedding noise source. For low Mach number flows (0.1884) and moderate to high chord Reynolds number, $4.73 \times 10^{5}-3.35 \times 10^{6}$, change in sound power level was assessed for trailing edge thicknesses in terms of $0.1 \%, 0.5 \%$ and $1 \%$ chord lengths at wind speeds of $8 \mathrm{~m} / \mathrm{s}, 10 \mathrm{~m} / \mathrm{s}$. For overall change of trailing edge thickness from $0.1 \%$ to $1 \%$ chord, an increase in noise levels up to $50 \mathrm{~dB}$ was found at low frequencies, while a decrease up to $30 \mathrm{~dB}$ was found between mid-band to high frequencies of spectra.
\end{abstract}

\section{Keywords:}

aerofoil, boundary layer, sound power level, wind turbine

\section{Introduction}

Wind turbine blades produce aerodynamic noise when they operate in varied terrains such as grass land, hills, or open sea surface. Aerodynamic noise produced from blades can be heard at audible frequencies which causes annoyance and sleep disturbance, as well as it creates health concerns, such as stress to inhabitants. Different community inhabitants across the world have mixed views regarding the presence of wind turbine operation located near their homes. Therefore, noise regulation standards are implemented in several countries, e.g. the UK, Germany, Denmark and Sweden where threshold outside environment noise level is $55 \mathrm{~dB}$ during day time and at $40 \mathrm{~dB}$ at night time, with a difference of $15 \mathrm{~dB}$ for urban mixed areas [1]. In the last century, the wind turbine sizes were predominantly smaller due to limited applications, however, in past few decades the size of modern wind turbines changed

\footnotetext{
* Corresponding author: GE Global Research, JFWTC, Whitefield, Bangalore, India. E-mail: vasishtab@gmail.com
} 
dramatically due to improvements in technology and design. The design of quieter wind turbine is highly dependent upon the tip speed of blade during operation.

Longer blades usually tend to possess high tip speed that acts as potential design constraint. It also drives the cost of energy for a given turbine since increased tip speed of blades tend to capture higher energy from a turbine, but it results in higher noise emissions. Aerodynamically produced noise utilizes the principles of classical acoustics and fluid mechanics and depends on the turbulent flow field characteristics over the moving surfaces, e.g. aerofoil. Broadband noise from wind turbines blades is caused due to the interaction of turbulent boundary layer flow over the trailing edge of blade. From many wind tunnel studies conducted on symmetric aerofoils in open circuit anechoic wind tunnel, a logarithmic relationship for sound pressure level was derived [2-5]. Further on, these studies also revealed that sound pressure level is affected by trailing edge thickness of blade and sloping angle between the trailing edge surfaces. A regression-based curve fitting relationship between the trailing edge height and turbulent boundary layer thickness was derived. This relationship describes the vortex shedding mechanism and was demonstrated by several experiment studies [2, 18 ] in an attempt to quantify noise radiation. The turbulent boundary layer flow downstream of trailing edge produces starting vortex, formed as a result of increasing boundary layer thickness due to velocity gradients along the chord line of aerofoil. Therefore, boundary layer thickness and displacement thickness contribute to noise radiation. It must be noted that sound pressure level as perceived by human ear varies with atmosphere conditions, as well as with the distance between the source and the receiver in far field. A better description of far-field sound levels is done using an inverse square relation between source and observer. The objective of this paper is to predict the far-field broadband and tonal noise caused due to trailing edge thickness of the aerofoil based on the method developed by Brooks Pope and Marcolini (BPM). For the present study, a $2 \mathrm{MW}$ wind turbine blade with the blade length of $37 \mathrm{~m}$ and the hub height of $\sim 80 \mathrm{~m}$ was considered to assess the sound power level at three trailing edge heights viz. $0.1 \%, 0.5 \%, 1 \%$ and $1.5 \%$ chord length. The effect of trailing edge thickness and bluntness parameters on the sound levels are illustrated for different free-stream flow conditions. The significance of the blade geometry and flow variables are investigated and regarded as important factors in the estimation of the strength of this source $[2-4,6]$. The flow field over the blade is computed using boundary element momentum (BEM) method to derive the angle of attack, inflow angles, blade relative velocity and coupled to noise solver. In addition, this work also aims to analyse the extent of reduction or increment in 1/3 octave band A-weighted sound power levels in sound spectrum as result of change in the trailing edge thickness.

\section{Method Description}

\subsection{BPM Model - Trailing Edge Bluntness Vortex Shedding}

The wind turbine blades experience subsonic or low Mach number flows and operate in atmospheric boundary layers where the effects of air density and wind shear on sound pressure levels are important. The noise from wind turbine blades depends upon aerofoil geometry, local angle of attack for the aerofoils, and rotational speed of the turbine. One of the noise mechanisms from blades is caused due to periodic vortex shedding from suction side of trailing edge surface and it exhibits tones in the sound spectrum. 
According to this model, the vortex shedding occurs when the boundary layer displacement thickness is at least 30\% higher than characteristic dimension of source $[2,3]$. For a given angle of attack, the vortex shedding from blunt trailing edge is caused due to the excessive adverse pressure gradient on suction surface of aerofoil. However, as the thickness of trailing edge increases, the boundary layer instabilities occur at faster rate which leads to the flow separation and it contributes to sound levels. According to this method, the strength of this source is approximated using the spectral functions, $G_{4}$ and $G_{5}$ which are functions of ratio of trailing edge thickness and average boundary layer displacement thickness from pressure and suction sides of aerofoil as given by Eq. (5). Hence, it is needed to compute the spectral functions $G_{4}$ and $G_{5}$ as given by Eq. (6)-(8). $G_{4}$ represents the narrowband peak in spectra and $G_{5}$ is used to determine the broadband overall shape of spectra which is dependent on Strouhal number, $S t$ "' and $S t_{\text {peak }}$ ".".

The spectral functions $\left(G_{5}\right)_{\varphi=14^{\circ}}$ and $\left(G_{5}\right)_{\varphi=0^{\circ}}$ are solid angles determined using the symmetric NACA 0012 aerofoil experiments and determined by Eq. from (76) to (82) given in [2]. As mentioned in [2], this source appears as tonal peak and contributes to the noise level near $10 \mathrm{kHz}$. The functional parameters in Eq. (1) are expressed in terms of the flow angle of attack, bluntness ratio $h / \delta^{*}$, for aerofoil at moderate to high Reynolds number; at the same time, they show the dependence of Mach number, $M^{5.5}$. The sound pressure levels are found to vary with the span segment length of aerofoil, $L$ and inverse square of the distance between source and receiver, $r^{2}$ as given in Eq. (1) [2]. The Strouhal number for this type of source is defined according to Eq. (2) where $h$ is the height of trailing edge. It must be noted that at moderate Reynolds number and for subsonic Mach number flows, the chord Reynolds number and boundary layer, displacement thicknesses for zero and non-zero angle of attack are evaluated using Eq. (5) and Eq. (16) [2]. The 1/3 octave sound pressure for this source is approximated using the Eq. (1). The narrowband tonal peak is given by function $G_{4}$ and expressed using Eq. (6) and Eq. (7).

Function $G_{5}$ is calculated using ratio of trailing edge thickness to average boundary layer displacement thickness and sloping angle, $\varphi$ between $0^{\circ}$ to $14^{\circ}$ given by Eq. (78) and Eq. (79) found in [1] where $\varphi$ is the angle between the sloping surfaces near trailing edge of aerofoil and $\delta^{*}$ and $\delta^{*}$ s are the pressure and suction side boundary layer displacement thickness, and $h$ is the trailing edge height. The empirical equations used to determine the pressure and suction side displacement thicknesses for zero and non-zero angle of attack for symmetric aerofoils are taken $[2,4,5]$. They are found to be dependent upon the local angle of attack and chord Reynolds number. For an aerofoil, it is expressed in terms of the boundary layer displacement thicknesses for the pressure and suction side. This source also uses the high frequency directivity function like turbulent boundary layer trailing edge noise and given by the Eq. (9).

$$
\begin{gathered}
S P L_{\text {Blunt }}=10 \log \frac{h M^{5.5} L D_{\mathrm{h}}}{r_{\mathrm{e}}^{2}}+G_{4}\left(\frac{h}{\delta_{\text {avg }}^{*}}, \varphi\right)+G_{5}\left(\frac{h}{\delta_{\text {avg }}^{*}}, \varphi, \frac{S t^{\prime \prime \prime}}{S t_{\text {peak }}^{\prime \prime \prime}}\right), \\
S t^{\prime \prime \prime}=\frac{f h}{U},
\end{gathered}
$$




$$
\begin{gathered}
S t_{\text {peak }}^{\prime \prime \prime}=\frac{0.212-0.0045 \varphi}{1+0.235\left(\frac{h}{\delta_{\text {avg }}^{*}}\right)^{-1}-0.0132\left(\frac{h}{\delta_{\text {avg }}^{*}}\right)^{-2}}, \text { for } \frac{h}{\delta_{\text {avg }}^{*}} \geq 0.2, \\
S t_{\text {peak }}^{\prime \prime \prime}=0.1\left(\frac{h}{\delta_{\text {avg }}^{*}}\right)+0.095-0.00243 \varphi, \text { for } \frac{h}{\delta_{\text {avg }}^{*}}<0.2, \\
G_{4}\left(\frac{h}{\delta_{\text {avg }}^{*}}, \varphi\right)=17.5 \log \frac{h}{\delta_{\text {avg }}^{*}}+157,5-1.114 \varphi, \quad \text { for } \frac{h}{\delta_{\text {avg }}^{*}} \leq 5, \\
G_{4}\left(\frac{h}{\delta_{\text {avg }}^{*}}, \varphi\right)=169.7-1.114 \varphi, \quad \text { for } \frac{h}{\delta_{\text {avg }}^{*}}>5, \\
G_{5}\left(\frac{\delta_{\mathrm{p}}^{*}+\delta_{\mathrm{s}}^{*}}{\delta_{\text {avg }}^{*}}, \varphi, \frac{S t^{\prime \prime \prime}}{S t_{\text {peak }}^{\prime \prime \prime}}\right)=\left(G_{5}\right)_{\varphi=0^{\circ}}+0.0714 \varphi\left[\left(G_{5}\right)_{\varphi=14^{\circ}}-\left(G_{5}\right)_{\varphi=0^{\circ}}\right], \\
D_{H}(\theta, \phi)=\frac{2 \sin { }^{2}(1 / 2 \theta) \sin ^{2} \phi}{(1+M \cos \theta)\left[1+\left(M-M_{\mathrm{C}}\right) \cos \theta\right]^{2}},
\end{gathered}
$$

where $\theta, \phi$ are the directivity angles between the source and receiver line aligned to blade span and chord direction with respect to the receiver position. $M$ is the Mach number and $M_{\mathrm{c}}$ is the convective Mach number. The denominator term in Eq. (9) represents the Doppler effect and convective amplification of acoustic waves produced at the trailing edge of aerofoil [2-4, 7, 8]. It has been proven that for high values of Strouhal number or for the order greater than 2, the flow is dominated by boundary layer thickness and results in small scale flow instabilities $[2,9,10]$.

The Strouhal number and the shape functions vary with the shape of aerofoil, inflow velocity conditions and local angle of attack. Experiments conducted by Brookes et al. [2] used a reference chord length for test aerofoil which was $30.86 \mathrm{~cm}$ and boundary tripping was done with help of $2 \mathrm{~cm}$ wide strip or grit applied at $15 \%$ chord length. Tripping of boundary layer resulted in reduction of the noise levels in certain regions of sound spectrum $[4,5,11]$. For the present analysis, tripping of boundary layer has not been taken into consideration.

The maximum trailing edge height in BPM model aerofoil was $2.5 \mathrm{~mm}$ which is $\sim 0.8 \%$ of chord. For the present case of $37 \mathrm{~m}$ blade, it is $3.22 \mathrm{~mm}, 16.1 \mathrm{~mm}, 32.2 \mathrm{~mm}$ and $48.3 \mathrm{~mm}$ and corresponds to $0.1 \%, 0.5 \%, 1 \%$ and $1.5 \%$ chord, respectively. Also for a blade length of $37 \mathrm{~m}$, the mean trailing edge angle of $5.6^{\circ}$ is evident as depicted in Fig 1(a) and maximum chord length of blade is $3.22 \mathrm{~m}$ for the turbine.

\section{Discussion}

In the analysis of sound pressure level from wind turbine blade, boundary element method was used to compute the relative velocity field along the blade span. The total length of blade is discretized into aerofoil segments typically in the order of 15. Aero- 
foil is modelled as half-infinite flat plate with finite thickness [2] and the flow over it is assumed to be $2 \mathrm{D}$ incompressible and quasi uniform along the blade length which means that there is no dependence of the span-wise correlation of the flow. The shape of blade is approximated using selected aerofoils, viz. NACA 0012, NACA 6320 and NACA 63215 and hence, the boundary layer properties on suction and pressure side of aerofoils along the span. The boundary layer data for individual aerofoils is obtained from X-Foil computations.

In the prediction of sound pressure levels, each blade segment is treated as a point source in near field and rotating blade as line source. In the far field however, the blades act as point source where the sound waves propagate or spread themselves in spherical manner. Sound pressure level is thus calculated by logarithmic addition of individual sources relative to observer position. For the present simulation work, the receiver height was fixed at $2 \mathrm{~m}$ above the ground level and the source height was fixed at $80 \mathrm{~m}$. The distance of the receiver location was set at $110 \mathrm{~m}$, which is approximately the total turbine height $(H H+D / 2)$. This is in accordance with IEC 61400-11 regulations for measurements of acoustic emissions from wind turbines. $H H$ is the hub height of turbine, and $D$ is the rotor diameter in [m].

A downwind scenario is considered as the worst case due to the fact that sound waves bend in downward direction with respect to free stream wind and this results in amplification. Therefore, downwind receiver location is taken into account. Tab. 1 shows the turbine configuration used in the computational study and Fig. 1(a) shows the chord and twist distribution for the rotor blade of $37 \mathrm{~m}$. Fig. 1(b) represents a 37 m-blade developed using open source software NuMAD by providing blade chord and twist parameters as input [12].

The boundary conditions for the blade are Reynolds number, the angle of attack along the blade span. It is implemented to verify that boundary element method (BEM) computed values do not exceed predefined threshold values as given in [2, 8]. The blade pitch angle is set to $3.5^{\circ}$ for sound pressure calculations and rotation speed for machine as $17 \mathrm{rev} / \mathrm{min}$.

Tab. 1 Turbine parameters for $2 \mathrm{MW}$ machine

\begin{tabular}{|l|c|}
\hline Parameter & Value \\
\hline Cone angle & $0^{\circ}$ \\
\hline Tilt angle & $3^{\circ}$ \\
\hline Hub height & $80 \mathrm{~m}$ \\
\hline Blade Radius & $37 \mathrm{~m}$ \\
\hline Rotor speed & $17 \mathrm{rev} / \mathrm{min}$ \\
\hline Max twist & $13^{\circ}$ \\
\hline Max chord & $3.22 \mathrm{~m}$ \\
\hline Orientation & Upwind \\
\hline No of blades & 3 \\
\hline Rated power & $2 \mathrm{MW}$ \\
\hline
\end{tabular}

The relative position of the receiver with respect to aerofoil coordinate system is shown in Fig. 2(a). The vortex shedding from trailing edge of an aerofoil is shown in Fig. 2(b). It reveals the significance of trailing edge thickness for a given set of flow conditions. For a wind turbine blade, in addition to the shear within turbulent boundary layer responsible for trailing edge vortex shedding noise, the sound levels also depend on blades pitch angle operation. Particularly for moderate pitch angles and at 
low or positive angle of attack, the boundary layer on the pressure side of aerofoil at leading edge shows laminar flow structure; however, the boundary layer on suction side remains mostly in turbulent state near the trailing edge. Further, it is important to note that such a type of noise mechanism is dominant in mid span region of blade where trailing edge thicknesses are high for which maximum Strouhal number is found to be 0.15 . Below this value, the vortex shed from the trailing edge does not contribute significantly to the sound levels $[3,4]$.

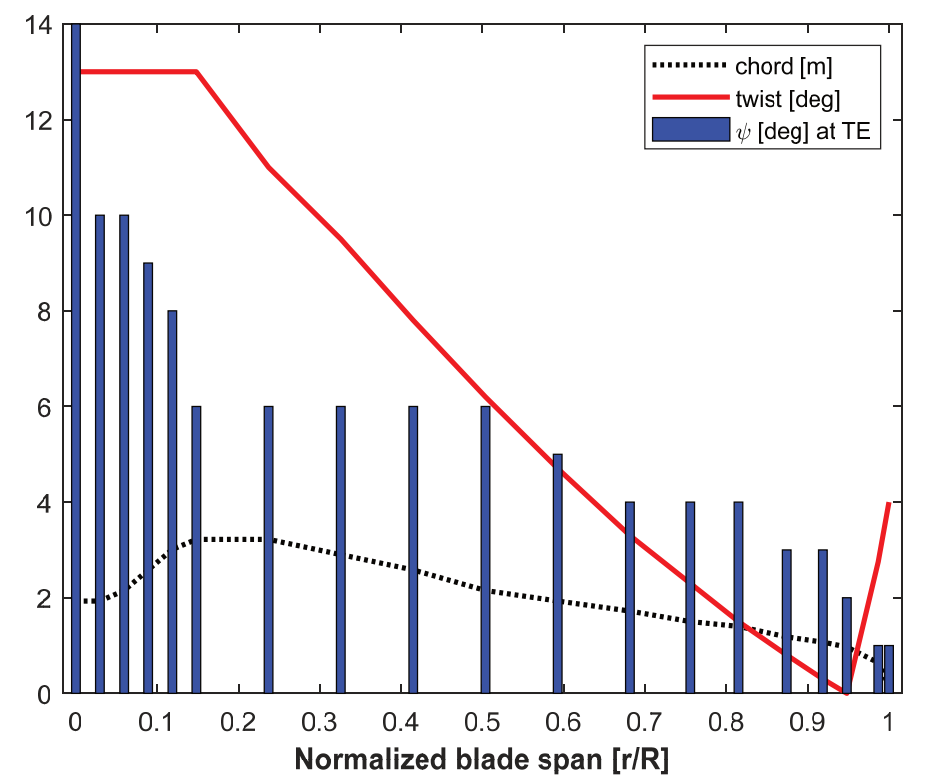

(a)

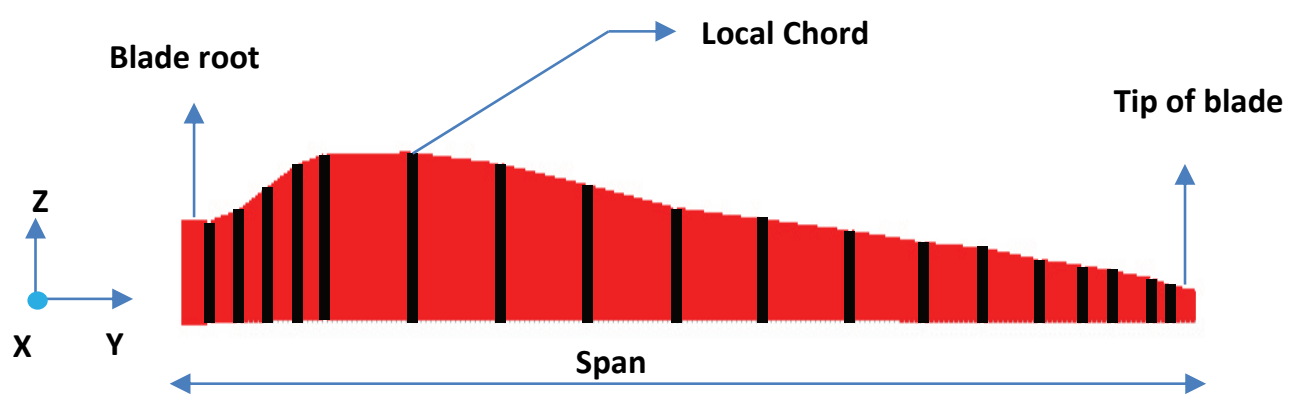

(b)

Fig. 1 (a) Geometric properties of $2 \mathrm{MW}$ wind turbine blade (b) Top view of $37 \mathrm{~m}$ blade developed using NuMAD software [12] 


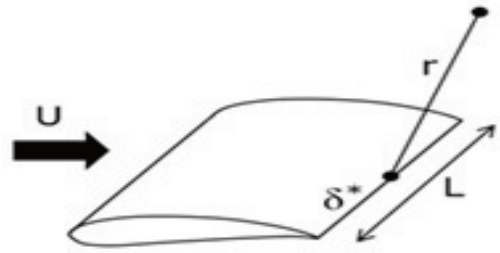

(a)

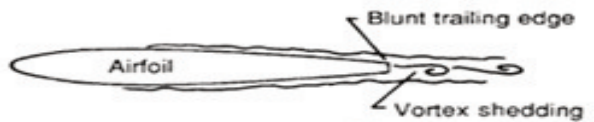

(b)

Fig. 2 (a) Illustration of distance between the source and receiver [2]

(b) Vortex shedding pattern from blunt trailing edge of an aerofoil [2]

\section{Results}

\subsection{Trailing Edge Thickness Effect}

The receiver position is placed at the distance of $2.2 R$ from the turbine at a ground height of $\sim 2 \mathrm{~m}$ where $R$ is the blade radius. The type $\mathrm{I}$ in the matrix uses the trailing edge thickness of $0.1 \%$ chord length and type II uses the trailing edge thickness of $0.5 \%$ chord length of aerofoils. For all the test cases in the matrix, computer simulations were performed using program code developed in MATLAB 2018b software. Tab. 2 shows the maximum values obtained from the simulated cases for three different wind speeds.

Fig. 3(b) illustrates the rotor azimuth averaged and blade averaged values obtained for vortex shedding noise at free stream wind speeds of $8 \mathrm{~m} / \mathrm{s}, 10 \mathrm{~m} / \mathrm{s}, 15 \mathrm{~m} / \mathrm{s}$ and $20 \mathrm{~m} / \mathrm{s}$. The maximum sound power levels for type I were found to be $113 \mathrm{~dB}$ and for type II it was $135 \mathrm{~dB}$ respectively. The chord Reynolds number which corresponds to the wind speeds is $1.3 \times 10^{6}, 2.6 \times 10^{6}, 4.87 \times 10^{6}$. The Strouhal number in the range of 0.01 and 10 was obtained for the test cases over the entire frequency region.

Tab. 2 Comparison of maximum sound power levels $[\mathrm{dB}]$

\begin{tabular}{|c|c|c|c|c|}
\hline \multirow{2}{*}{$\begin{array}{c}\text { TE thickness } \\
\text { [\%] chord }\end{array}$} & \multirow{2}{*}{ Type } & \multicolumn{3}{|c|}{ Wind speed [m/s] } \\
\cline { 3 - 5 } & & 8.00 & 10.00 & 15.00 \\
\hline 0.1 & I & 86.84 & 96.18 & 116.12 \\
\hline 0.5 & II & 103.86 & 115.01 & 135.26 \\
\hline
\end{tabular}

It is evident from Fig 3(a) that by decreasing the trailing edge thickness, the bluntness noise contribution will reduce by $5 \mathrm{~dB}$. A broad shape of spectrum is observed for the thickness of $0.5 \%, 1 \%$ and $1.5 \%$ chord length, near $1 \mathrm{kHz}$ caused due to the higher boundary layer thickness leading to vortex shedding from suction side of aerofoil and at $2 \mathrm{kHz}$, a smaller tone is also evident which is produced from the pressure side of aerofoil. Further from Fig 3(a) the tonal peak in sound spectrum for $0.1 \%$ chord length is found at $10 \mathrm{kHz}$. Therefore, at lower thickness and bluntness angle, this type of noise does not show significant variations other than the peak in the sound spectrum, i.e. $f<5 \mathrm{kHz}$. The maximum reduction of sound power levels between the type I and type II test matrix are found to reach $\sim 35 \mathrm{~dB}$ observed between $2 \mathrm{kHz}$ and 


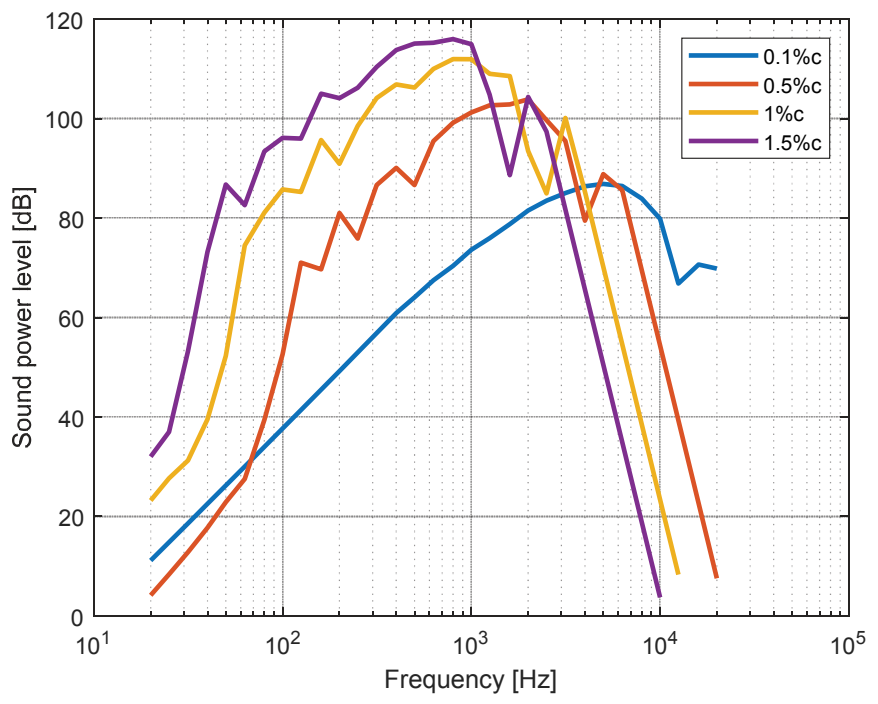

(a)
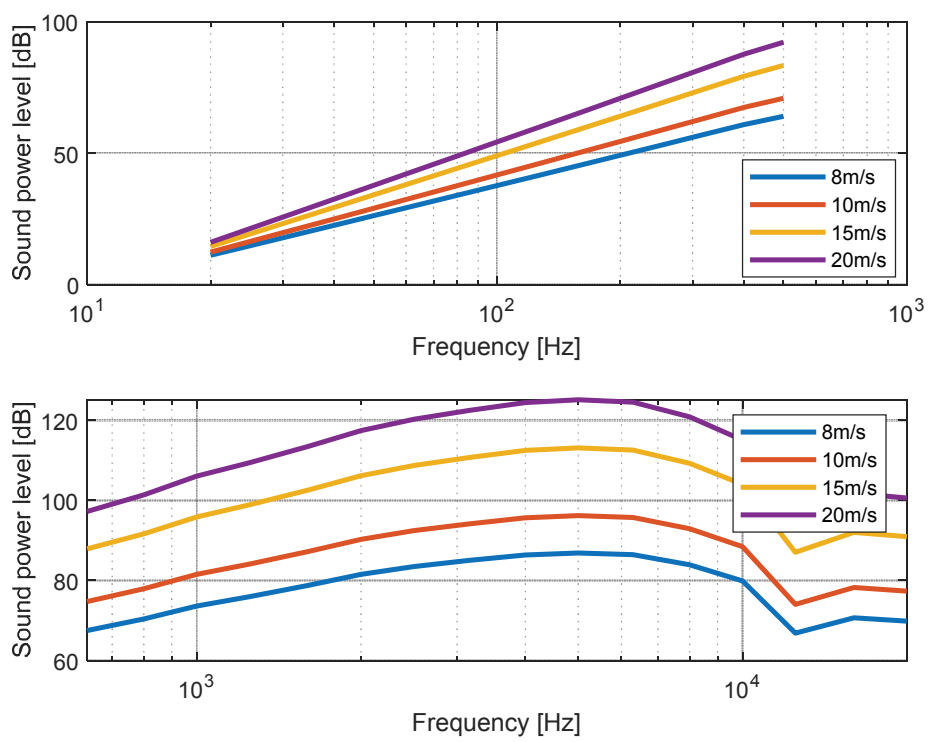

(b)

Fig. 3 (a) Influence of trailing edge thickness on sound power level from vortex shedding noise $[\mathrm{dB}]$ at hub height wind speed of $8 \mathrm{~m} / \mathrm{s}(b)$ Rotor azimuth averaged and blade averaged vortex shedding noise $[\mathrm{dB}]$ values for TE-thickness of $0.1 \%$ chord at wind speeds of $8 \mathrm{~m} / \mathrm{s}, 10 \mathrm{~m} / \mathrm{s}, 15 \mathrm{~m} / \mathrm{s}$ and $20 \mathrm{~m} / \mathrm{s}$ 
$8 \mathrm{kHz}$. In addition to blade characteristics, the physics of the vortex generation from an aerofoil is governed by the chord Reynolds number, the angle of attack and stall on blade surface. The stall phenomenon is observed between $12^{\circ}$ and $16^{\circ}$ angles of attack for most of NACA aerofoils and it contributes to sound levels [5]. Previous studies have also shown that active flow control over aerofoils with porosity have the potential to reduce noise significantly due to control of boundary layer thickness on surface $[6,13,14]$. These methods are used to energize the flow that can withstand adverse pressure gradient and delay the flow separation over the trailing edge surface of aerofoil [15].

\subsection{Sound Level Change}

From Fig. 4(a) it can be seen that noise increments are observed for frequencies $f<2 \mathrm{kHz}$ and decrease in noise levels is observed for frequencies $f>5 \mathrm{kHz}$. Further the noise reductions are achieved for change in trailing edge thickness from $0.5 \%$ to $0.1 \%$ chord producing tonal peak. The tonal peak in spectrum vary with Strouhal number and exhibit high sound intensity level. As mentioned in section 2, the amplitude of tone noise is affected by the thickness of trailing edge and trailing edge angle. Further, at low to moderate frequencies between $100 \mathrm{~Hz}$ and $1100 \mathrm{~Hz}$, the noise level is increased and appears as tones. The tones are found at $200 \mathrm{~Hz}$ in spectrum and reach values up to the order of $35 \mathrm{~dB}$. On the contrary, for high frequencies between $4.5 \mathrm{kHz}$ and $10 \mathrm{kHz}$, the trailing edge bluntness noise tend to decrease sound power level, in the order of $20 \mathrm{~dB}$. Similarly to Fig 4(b) for a change of trailing edge thickness from $0.5 \%$ to $1 \%$ chord, the increment of sound levels is found to be as high as $50 \mathrm{~dB}$ between $100 \mathrm{~Hz}$ and $1.5 \mathrm{kHz}$, but it continues to decrease in high frequency region. In Fig 4(a) and Fig 4(b), a negative value indicates the reduction while positive values show an increase in noise levels. Hence, it is evident that the influence of bluntness parameters shows tonal peaks in spectrum. At high wind speeds, $U>U_{\text {rated, }}$, this effect is less pronounced than at below rated wind speeds i.e. $U<U_{\text {rated }}$ and it does not exhibit the large tonal peak found in high-frequency region. Also, for a given flow condition, the boundary layer displacement thickness on the aerofoil surface is found to develop more slowly at high Reynolds number and to extend along the chord direction of blade. The development of boundary layer thickness over aerofoil surface is attributed to the strength of pressure gradient within the boundary layer. An adverse pressure gradient appears when the boundary layer thickness on the surface of aerofoil exceeds a critical value known as point of inflexion. Beyond this point, the flow separates and extends as turbulent vortex flow downstream of the trailing edge. It must be noted here that viscous nature of turbulent boundary layer on the trailing edge region and its interaction with aerofoil surface is responsible for edge scattering and it leads to subsequent noise radiation [2-4, 8,9]. The acoustic waves radiated near trailing edge come in contact with incoming flow field along the chord. The sound waves in this region are also believed to travel backwards to the leading edge of aerofoil through a feedback mechanism which is caused due to the formation of T-S (TollmeinSchlichting) oscillating waves in flow field and observed in laminar boundary layer region near leading edge of aerofoil. The perturbed waves of incoming flow travel again downstream along the chord direction towards trailing edge of aerofoil. Studies have shown that the exact reason behind this feedback mechanism is not known for certain and it is beyond the scope of the present study [10]. 
For the trailing edge thickness of $0.1 \%$, the narrow peak of sound power level is observed at $10 \mathrm{kHz}$ and it is attributed to vortex shedding from trailing edge of blade. The nature of vortex shedding from the trailing edge depends on dominant frequency in sound spectrum defined by Strouhal number, as well as by Reynolds number. This frequency is found to vary with the magnitude of free stream velocity and the turbulent boundary layer displacement thickness [2, 3, 8]. The broad shape of spectrum however decreases gradually and results in truncated form at high frequencies in spectrum, $f>5 \mathrm{kHz}$. Further, the amplitude of sound level reaches the peak between $5 \mathrm{kHz}$ and $10 \mathrm{kHz}$. This peak extends to $10 \mathrm{kHz}$ at which the sound power level is found to be $91 \mathrm{~dB}(\mathrm{~A})$ for wind speed of $10 \mathrm{~m} / \mathrm{s}$. From Fig. 6(b), for frequencies below $10 \mathrm{kHz}$, the experimental data for GE-5sle turbine with $77 \mathrm{~m}$ rotor diameter show good agreement within $1 \%$ to the computational results obtained for the $2 \mathrm{MW}$ turbine. The trailing edge thickness effect on the overall sound power level (OASPL) measurements is also seen negligible for experiment data obtained for GE-1.5sle machine with hub height of $80 \mathrm{~m}$. Also from Fig. 4(a) and Fig. 4(b) it can be seen that sound power levels begin to reduce for mid-band to high frequency region of spectrum, for $f>1.5 \mathrm{kHz}$ when the trailing edge height is increased while for low frequency region of spectrum, for $\mathrm{f}<1 \mathrm{kHz}$ an increase in sound power levels are found. This study also confirms the results obtained from experiment work of Brooks and Hodgson [18]. Experiements from [18] have also revealed that the trailing edge bluntness noise source showed high sound amplitudes for increasing values of trailing edge heights, $h / \delta^{*}$. In their experiments, NACA 0012 aerofoil was used with thickness to chord ratio $(t / c)$ of 0.12 , chord length of $60.96 \mathrm{~cm}$ and span of $46 \mathrm{~cm}$. The trailing edge thickness varied between $0 \mathrm{~mm}$ and $2.5 \mathrm{~mm}$. In the present study however, the maximum thickness corresponds to $1.5 \%$ of blade chord length and equals $48.3 \mathrm{~mm}$.

Experimental and theoretical work conducted by Howe [19] and Glegg et al [20] claim that as thickness of aerofoil is increased, noise reductions for mid-band to high frequency region of spectrum and increase in noise levels in the low frequency region were found. These studies were also aimed at assessing the noise reductions from the trailing edge surface of aerofoil by means of porous surfaces and trailing edge serrations as well as influence of angle of attack on aerofoil.

This confirms that the thickness of trailing edge along blade span show a direct relation with amplitude of sound levels and contributed mainly from mid span region of blade. Therefore, it can be concluded that trailing edge bluntness source masks other noise mechanisms at $10 \mathrm{kHz}$ region [2-5]. The Strouhal number variation is observed from 0.01 to 0.13 at free stream wind speeds $4 \mathrm{~m} / \mathrm{s}, 8 \mathrm{~m} / \mathrm{s}, 15 \mathrm{~m} / \mathrm{s}$ and $20 \mathrm{~m} / \mathrm{s}$ for the case of $0.1 \%$ chord. It can be noted that the peak of sound power level is found to increase by $5 \mathrm{~dB}$ due to the increase in wind speed near $10 \mathrm{kHz}$. Also, for high trailing edge thickness ratios the tonal peak exhibits an oscillatory behaviour near low frequency region of spectrum.

Further, it is also evident that tonal peak appears only near higher frequencies when the trailing edge thickness is a function of $0.1 \%$ of the chord length. So, for low Mach number flows, given the higher bluntness ratio $\left(h / \delta^{*}\right)$, the tonal peak is found to shift to lower frequencies, as shown in Fig. 3(a). Fig. 5(a) and Fig 5(b) show the trailing edge thickness effect and influence of free stream velocities on the peak Strouhal number along normalized blade span. As the trailing edge height is increased, the peak Strouhal number increases, but it remains almost steady along the blade span for a given thickness case. On the contrary, with an increase in free stream velocities, the peak Strouhal number shows a linear increase in trend along the span-wise direction. 
Towards the tip, for all velocities, the peak Strouhal number approaches nearly the same value. It indicates that the overall sound power level from turbine is functionally varying with the angle of attack or otherwise blade pitch angle for a given flow condition.

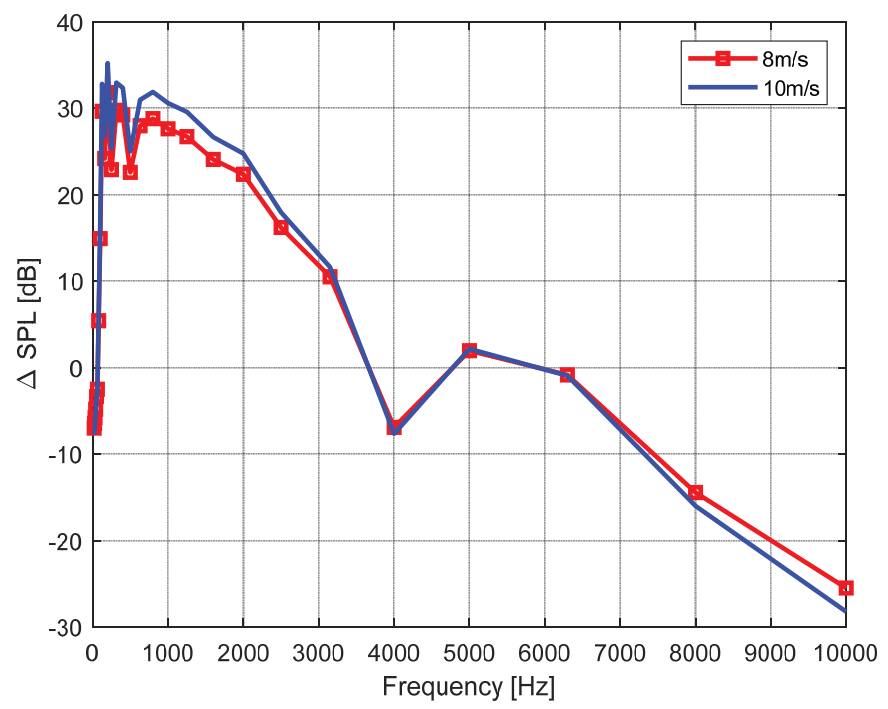

(a)

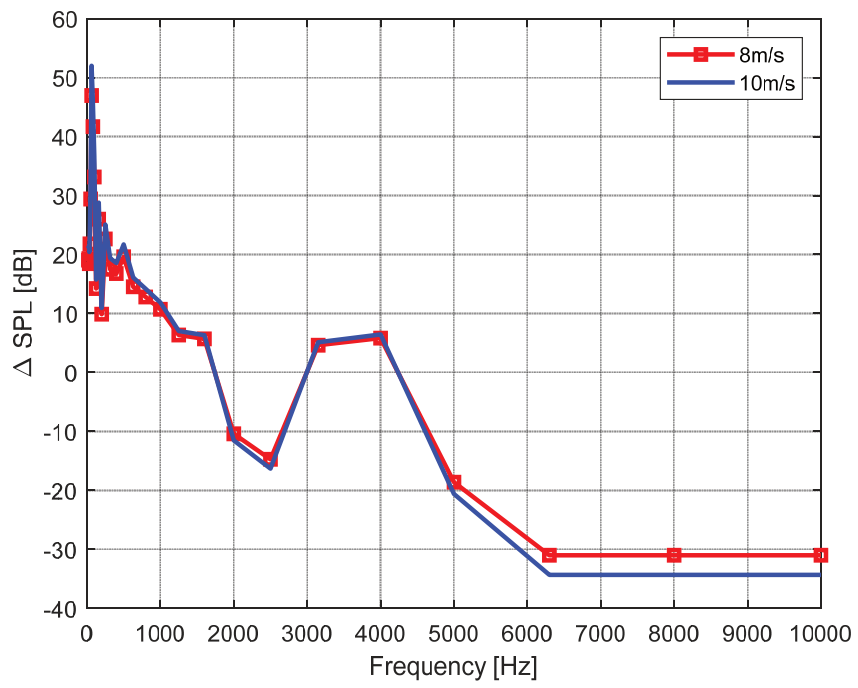

(b)

Fig. $4 \triangle S P L[\mathrm{~dB}]$ (a) for a change of trailing edge thickness from $0.1 \%$ to $0.5 \%$ chord at wind speeds of $8 \mathrm{~m} / \mathrm{s}$ and $10 \mathrm{~m} / \mathrm{s}(b)$ for a change of trailing edge thickness from $0.5 \%$ to $1 \%$ chord at wind speeds of $8 \mathrm{~m} / \mathrm{s}$ and $10 \mathrm{~m} / \mathrm{s}$ 


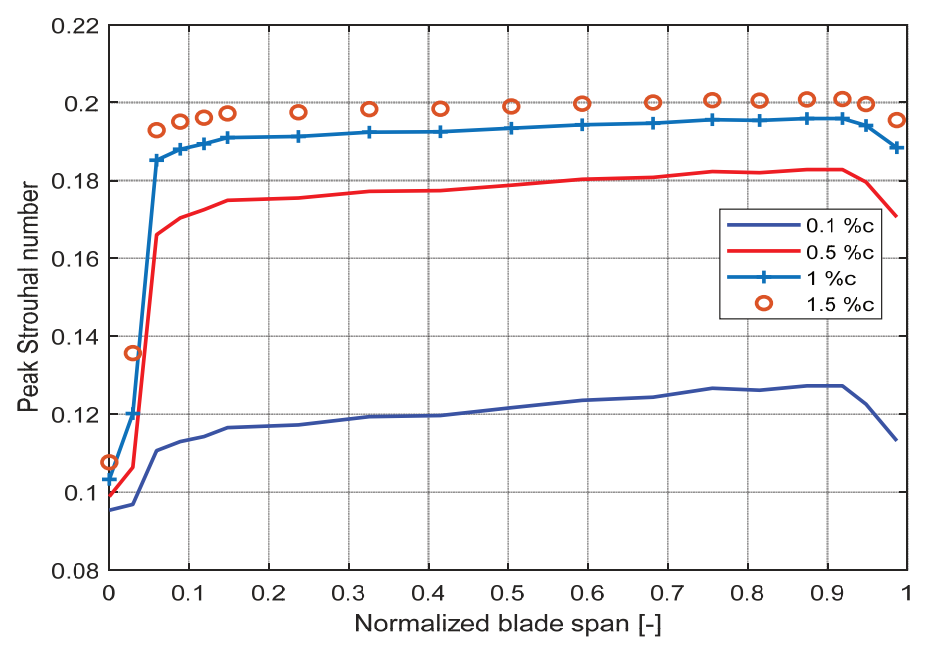

(a)

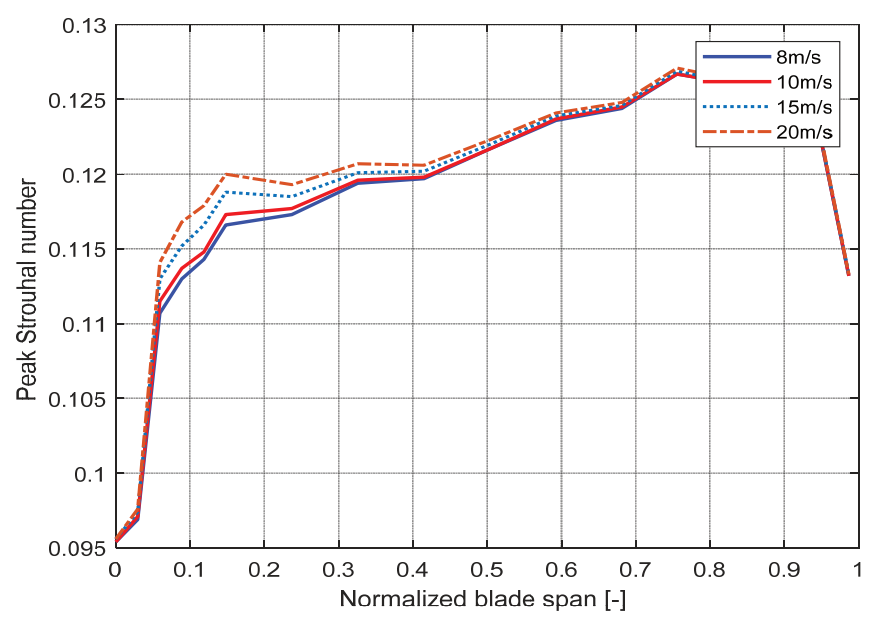

(b)

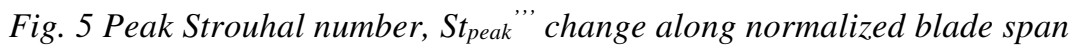

(a) for varying trailing edge heights, $0.1 \%, 0.5 \%, 1 \%$ \& $1.5 \%$ of chord length at $U=8 \mathrm{~m} / \mathrm{s}$ (b) for varying free stream velocities, $U=8 \mathrm{~m} / \mathrm{s}, 10 \mathrm{~m} / \mathrm{s}, 15 \mathrm{~m} / \mathrm{s}, 20 \mathrm{~m} / \mathrm{s}$ at TE thickness of $0.1 \%$ chord length

Fig. 6(b) shows that overall A-weighted sound power level for $2 \mathrm{MW}$ turbine agrees well with the experiment data obtained for GE-1.5sle turbine with $77 \mathrm{~m}$ rotor diameter [16]. The overall sound levels also include turbulent inflow noise mechanism proposed by Lowson [3-5]. It is based on Amiet's theory [3, 4, 8] for inflow turbulence noise prediction developed for thin symmetric aerofoils. It can be seen that maximum 
value of $95 \mathrm{~dB}(\mathrm{~A})$ and $105 \mathrm{~dB}(\mathrm{~A})$ is observed near $500 \mathrm{~Hz}$ region of spectrum and correlate very well with experiment data at $8 \mathrm{~m} / \mathrm{s}$ and $10 \mathrm{~m} / \mathrm{s}$.

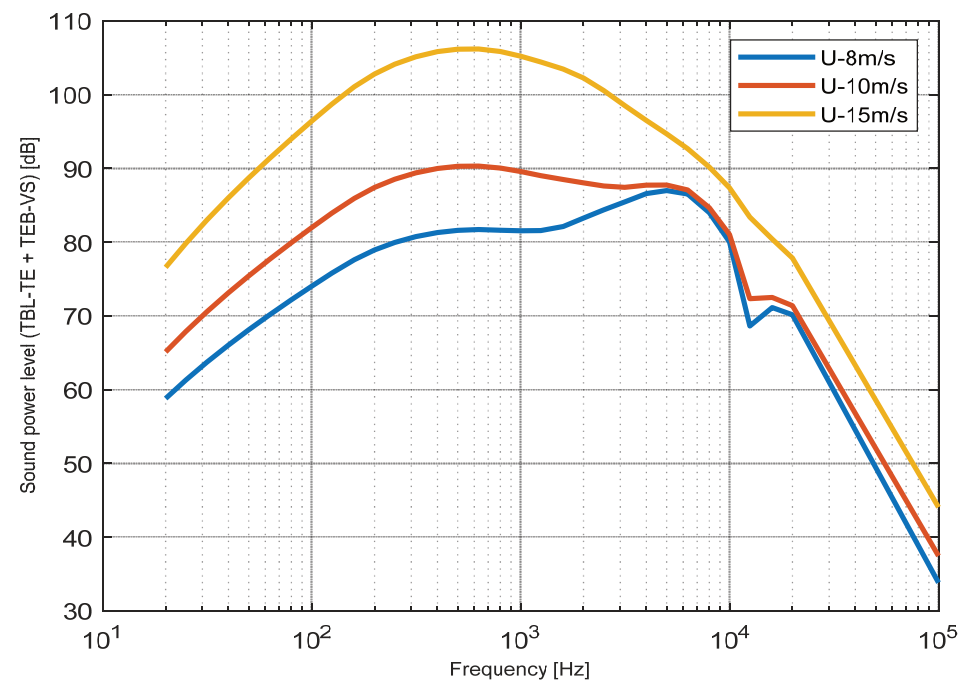

(a)

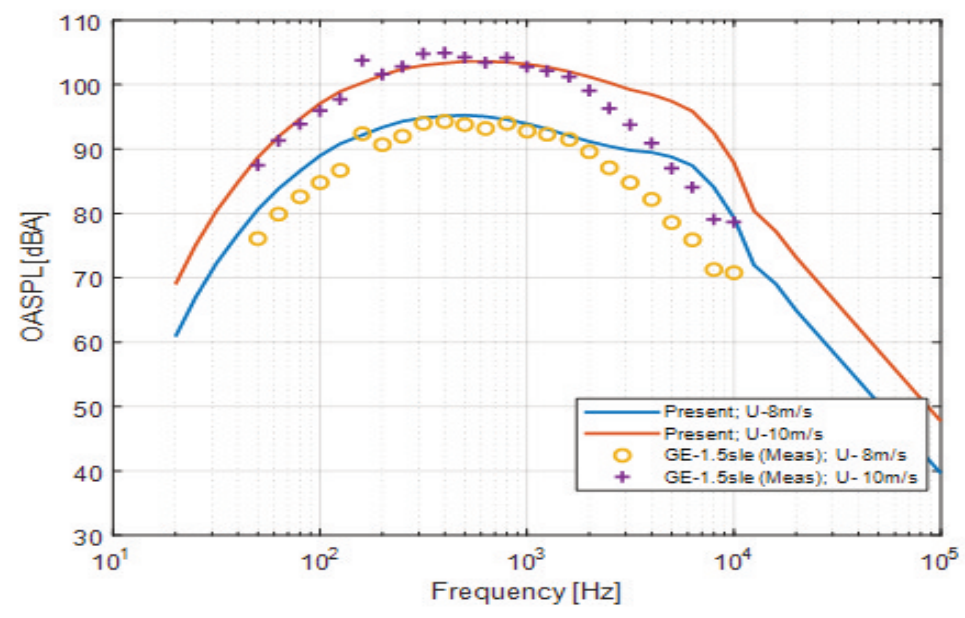

(b)

Fig. 6 (a) Sound power level, dB, due to combined effect from turbulent boundary layer trailing edge noise and trailing edge bluntness vortex shedding noise sources at free stream wind speeds of $8 \mathrm{~m} / \mathrm{s}, 10 \mathrm{~m} / \mathrm{s}$ and $15 \mathrm{~m} / \mathrm{s}$ using $0.1 \%$ trailing edge thickness $\left(h / \delta^{*}\right)(b)$ Validation of $1 / 3$ octave A-weighted overall sound power level for $2 \mathrm{MW}$ turbine of blade length $37 \mathrm{~m}$, with measured results of GE-1.5sle, turbine with $77 \mathrm{~m}$ rotor diameter using $0.1 \%$ trailing edge thickness $\left(h / \delta^{*}\right)$ 
Turbulence intensity and length scale are two important parameters in prediction of turbulent inflow noise $[3,4,17]$. So, in the present study, an integral length scale that represents a maximum aerodynamic chord length of blade is chosen. A turbulence intensity value of $14 \%$ is regarded as high according to IEC 61400-1 turbine design regulations. Turbulence intensity values change according to the type of terrain condition. Therefore, a high value is chosen for the present case which better represents the turbine operating environment or location, i.e. countryside or forest land and hence to predict the inflow noise level. Also from Fig. 6(a) for the above rated wind speeds, i.e. $15 \mathrm{~m} / \mathrm{s}$, the trailing edge bluntness noise source becomes less significant and it does not show tonal peak at high frequencies, $\sim 10 \mathrm{kHz}$ as found for wind speeds at $8 \mathrm{~m} / \mathrm{s}$ and $10 \mathrm{~m} / \mathrm{s}$. It can be inferred that for pitch controlled turbines, at the above-rated wind speeds, the change in the maximum sound levels is also found to vary negligibly as rotational speed of machine is kept constant. Therefore, the overall sound levels do not vary significantly. Fig. 7(a) to Fig 7(d) shows the contour plots of variation of the peak Strouhal number with the blade azimuth angle for free stream velocity of $8 \mathrm{~m} / \mathrm{s}$. Since the trailing edge heights vary along the blade span direction, the Strouhal number has been plotted against the normalized blade span direction

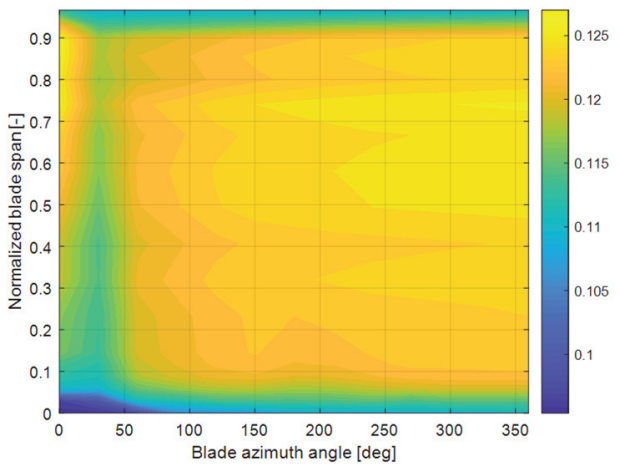

(a)

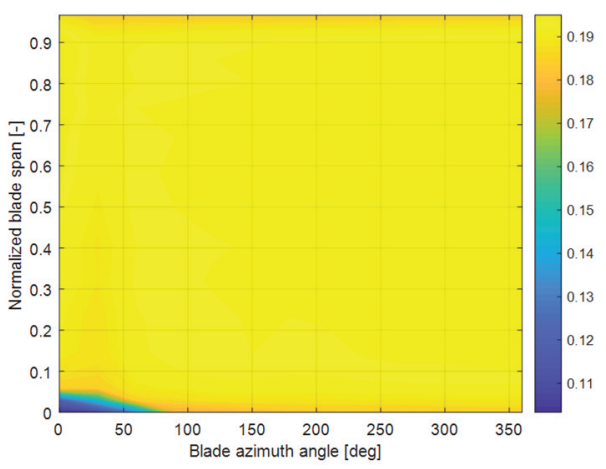

(c)

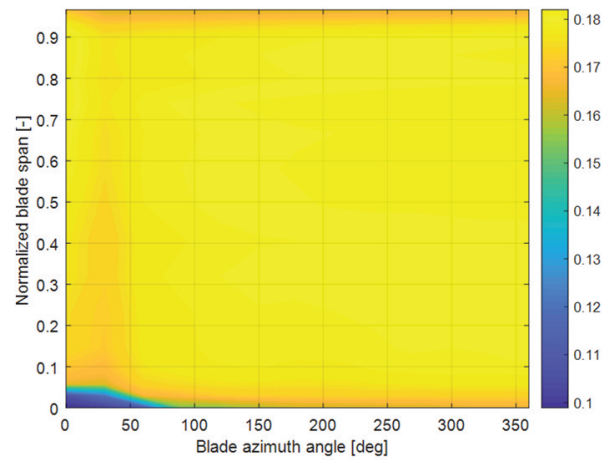

(b)

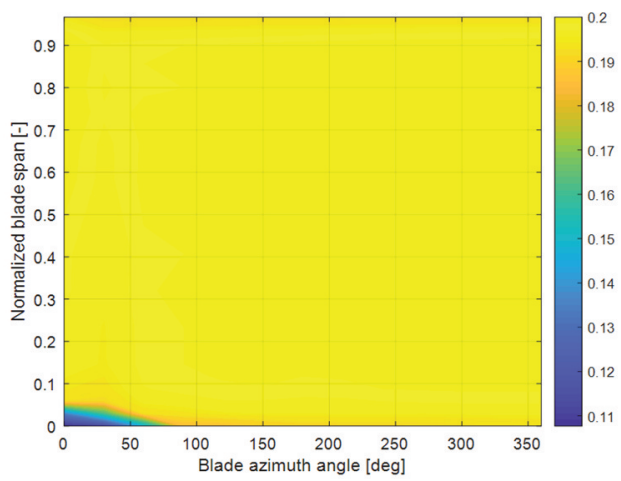

(d)

Fig. 7 Peak Strouhal number, Stpeak ,', along normalized blade span and blade azimuth angles at $U=8 \mathrm{~m} / \mathrm{s}$ for TE thicknesses (a) $0.1 \%$ chord (b) $0.5 \%$ chord 


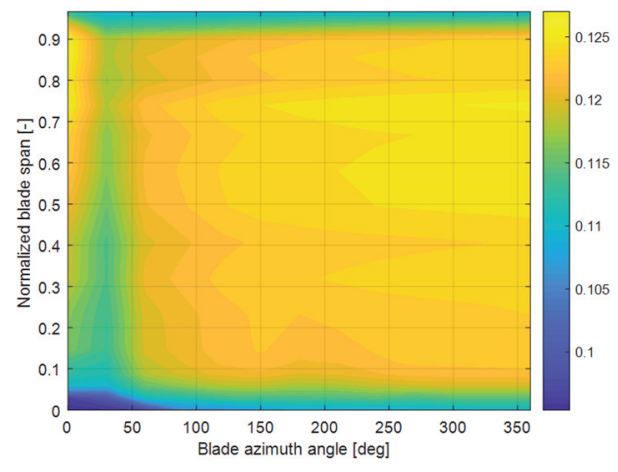

(a)

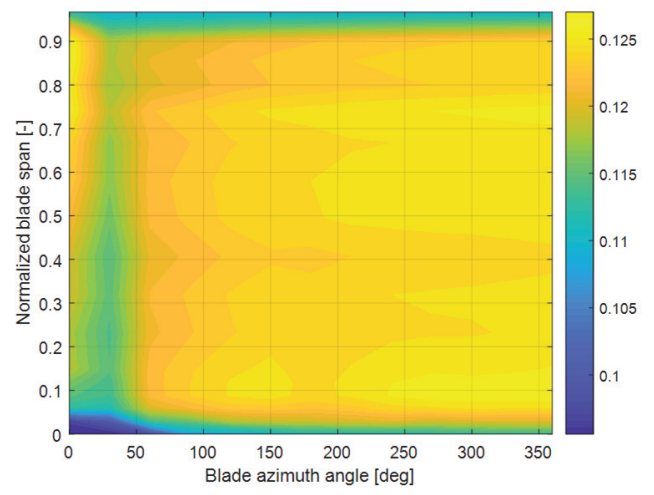

(c)

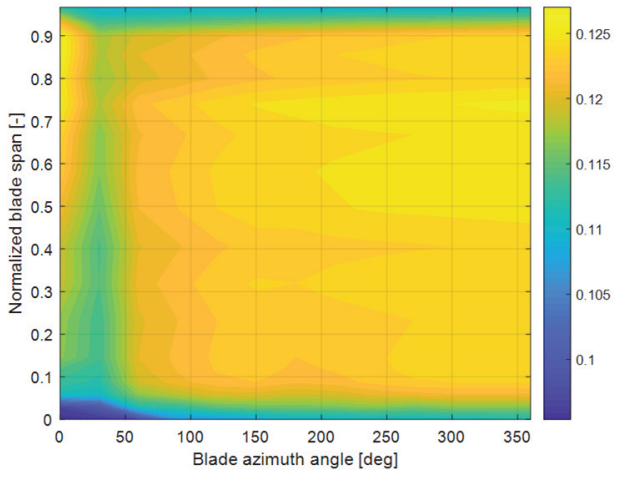

(b)

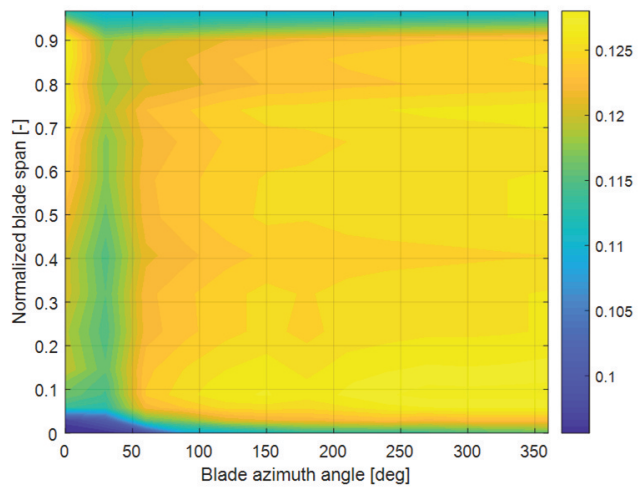

(d)

Fig. 8 Peak Strouhal number, St $t_{\text {peak }}$,", along normalized blade span and blade azimuth angles for trailing edge thickness of $0.1 \%$ chord length at (a) $U=8 \mathrm{~m} / \mathrm{s}$, (b) $U=10 \mathrm{~m} / \mathrm{s}$, (c) $U=15 \mathrm{~m} / \mathrm{s}$, (d) $U=20 \mathrm{~m} / \mathrm{s}$

Figs from 8(a) to 8(d) illustrate the peak Strouhal number that has been evaluated along the blade span for free stream velocities of $8 \mathrm{~m} / \mathrm{s}, 10 \mathrm{~m} / \mathrm{s}, 15 \mathrm{~m} / \mathrm{s}$ and $20 \mathrm{~m} / \mathrm{s}$, respectively. It can be noticed that Strouhal number varied significantly between $0.1 r / R$ and $0.5 r / R$ of blade span where the trailing edge bluntness ratio $\left(h / \delta^{*}\right)$ is high. However, towards the tip of blade, this change is found to vary negligibly irrespective of the wind speed. The maximum values for Strouhal number are found between 0.01 and 0.2. According to Fig. 6(b), the sound power level is dominated by the turbulent boundary layer trailing edge noise in nominal mid-band frequency range, i.e. $63 \mathrm{~Hz}$ and the $8 \mathrm{kHz}$ with approximately $95 \mathrm{~dB}(\mathrm{~A})$ at the wind speed of $8 \mathrm{~m} / \mathrm{s}$. In the low frequency region, however, between $60 \mathrm{~Hz}$ and $200 \mathrm{~Hz}$ turbulent inflow noise dominates the other noise mechanisms, i.e. turbulent boundary layer trailing edge (TBLTE) and turbulent boundary layer vortex shedding noise (TEB-VS). The experimental data for the GE 1.5 sle machine does not show the tonal peak effect near $10 \mathrm{kHz}$, as it is observed from computational prediction using BPM model. Hence, it confirms that trailing edge bluntness vortex shedding noise becomes an important noise source for wind turbines with an increase in trailing edge thickness of blade. 


\section{Conclusions}

A computational analysis of trailing edge bluntness vortex shedding noise mechanism for horizontal axis $2 \mathrm{MW}$ turbine was performed for different trailing edge thicknesses. For the trailing edge thickness of $0.1 \%$, chord the effect on $1 / 3$ octave overall A weighted sound level showed the tonal peak near $10 \mathrm{kHz}$ region and masks all other self-noise mechanisms. The existing experimental data showed very good agreement with simulated outputs for the 1/3 octave overall A-weighted sound power level spectrum at wind speed of $8 \mathrm{~m} / \mathrm{s}$ and $10 \mathrm{~m} / \mathrm{s}$. The increase in noise levels is observed up to $35 \mathrm{~dB}$ for a thickness increment from $0.1 \%$ to $0.5 \%$ chord length while for an increment of $0.5 \%$ to $1 \%$ chord length it is found to be up to $50 \mathrm{~dB}$ in the low frequency region of spectra, while a decrease of sound levels up $30 \mathrm{~dB}$ was found in the midband to high frequency region.

\section{Acknowledgement}

The authors would like to thank the organizations involved for providing the constructive comments received for the work. Special thanks to Prof. G Damodar, adjunct professor Satavahana University for English language proof-reading.

\section{References}

[1] BASTASCH, M., van DAM, J., SONDERGAARD, B. and ROGERS, A. Wind Turbine Noise - An Overview. Journal of Canadian Acoustical Association, 2006, vol. 34, no 2, p. 7-16.

[2] BROOKS, T.F., POPE, D.S. and MARCOLINI, M.A. Airfoil Self Noise and Prediction. NASA reference publication 1218, 1989, Available from: https://ntrs.nasa.gov/archive/nasa/casi.ntrs.nasa.gov/19890016302.pdf.

[3] GROSVELD, F.W. Prediction of Broadband Noise from Horizontal Axis Wind Turbines. Journal of Propulsion and Power, 1985, vol. 1, no 4, p. 292-299. ISSN 0748-4658.

[4] MORIARTY, P. and MIGLIORE, P. Semi Empirical Aero-Acoustic Noise Prediction Code for Wind Turbines [Technical report], 2003. 39 p. Available from:http://citeseerx.ist.psu.edu/viewdoc/download?doi=10.1.1.197.1153\&rep=re p1\&type $=$ pdf.

[5] ZHU, W.J. Modeling of Noise from Wind Turbines [Master thesis]. Lyngby: Department of Wind Energy, Technical University of Denmark, 2004, 105 p.

[6] DOOLAN, C., MOREAU, D.J., ARCONDOUliS, E. and ALBARRACIN, C. Trailing Edge Noise Production, Prediction and Control. New Zealand Acoustics, 2012, vol. 25, no 3, p. 22-29.

[7] BLANDEAU, V.P and JOSEPH, P.F. Validity of Amiet's Model for Propeller Trailing Edge Noise. AIAA Journal, 2011, vol. 49, no 5, p. 1057-1066. DOI 10.2514/1.J050765.

[8] DIJKSTRA, P. Rotor Noise and Aero-Acoustic Optimization of Wind Turbine Aerofoils [Master Thesis]. Delft: Delft University of Technology, 2015, $131 \mathrm{p}$.

[9] LELOUDAS, G. Optimization of Wind Turbines with Respect to Noise [Master Thesis]. Lyngby: Technical University of Denmark, 2006, 66 p. 
[10] BLAKE, W.K. Aero-Hydro-Acoustics for Ships Volume II [Technical Report] 1984. Available from: https://apps.dtic.mil/dtic/tr/fulltext/u2/a150672.pdf.

[11] MOREAU, D.J, BROOKS, L.A. and DOOLAN, C. Flat Plate Self-Noise Reduction at Low to Moderate Reynolds Number with Trailing Edge Serrations. In Proceedings of Acoustics. Gold Coast: Australia, 2011. Available from: https://pdfs.semanticscholar.org/74fa/0f9f258c0d5c5a1f846abb244c6d7505b213.pdf.

[12] NuMAD software, Version: NuMADexe_130403_PCWIN64. Available from: https://energy.sandia.gov/energy/renewable-energy/wind-power/rotor-innovation/ numerical-manufacturing-and-design-tool-numad.

[13] GEYER, T., SARRADJ, E. and FRITZSCHE, C. Porous Aerofoils: Noise Reduction and Boundary Layer Effects. International Journal of Aeroacoustics, 2010, vol. 9, no. 6, p. 787-820. DOI 10.1260/1475-472X.9.6.787.

[14] KINGAN, K.M. Aero-Acoustic Noise Produced by an Aerofoil [Doctoral thesis]. Christchurch: University of Canterbury, New Zealand, 2005. p. 448. Available from: https://ir.canterbury.ac.nz/handle/10092/6596.

[15] OJHA, C.S.P., BERNDTSSON, P.N. and CHANDRAMOULI, P. Fluid Mechanics and Machinery. Oxford: Oxford University Press, 2010. ISBN 0-19-569963-7.

[16] O’NEAL, R.D., ROBERT. D., HELLWEG, R.D. jr. and LAMPETER, R.M. A Study of Low Frequency Noise and Infrasound from Wind Turbines, 2009. 61 p. Available from: http://www.nexteraenergycanada.com/pdf/Epsilon_study.pdf.

[17] HIRSCHBERG, A. and RIENSTRA, S.W. An Introduction to Aeroacoustics [Technical report]. Eindhoven: University of Technology, 2004. 64 p. Available from: https://www.win.tue.nl/ sjoerdr/papers/les-swr-mh.pdf.

[18] BROOKS, T.F and HODGSON, T.H. Trailing Edge Noise Prediction from Measured Surface Pressures. Journal of Sound and Vibration, vol. 78, no. 1, p. 69-117. DOI 10.1016/S0022-460X(81)80158-7.

[19] HOWE, M.S Theory of Vortex Sound. Cambridge: Cambridge University Press, 2002. 232 p. ISBN 978-0-511-75549-1.

[20] GLEGG, S.A.L, DEVENPORT, .W.J. and STAUBS, .J.K. Sound Radiation from Real Airfoils in Turbulence. Journal of Sound and Vibration, 2010, vol. 329, no. 17, p. 3470-3483. DOI 10.1016/j.jsv.2010.02.022. 